\title{
Lacrimal sac diverticulum due to Rhinosporidiosis
}

\author{
Saurabh Varshney - S. S. Bist · Pratima Gupta · Nitin Gupta - Rajat Bhatia
}

\begin{abstract}
Rhinosporidiosis is a disease affecting primarily the mucosa of nose, conjunctiva and urethra. Larynx, trachea, skin and lungs are less frequently involved. It is endemic in some Asiatic regions, affecting people of any age and sex. Its manifestation is a polypoid mass growing inside the affected nasal cavity and the treatment is surgical excision, with adjuvant radiotherapy and chemotherapy. Rhinosporidium seeberi is the aetiological agent. The lesion may recur and sometimes cause osteolytic bone lesions. A case of Rhinosporidiosis with an unusual feature of spread to the nasopharyngeal area and chronicity of 2 years is presented.
\end{abstract}

Keywords Rhinosporidiosis · lacrimal sac $\cdot$ ocular rhinosporidiosis - Rhinosporidium seeberi

\author{
S. Varshney $(\bowtie) \cdot$ S. S. Bist ${ }^{2} \cdot$ P. Gupta $^{3} \cdot$ N. Gupta ${ }^{4} \cdot$ R. Bhatia ${ }^{5}$ \\ ${ }^{1}$ Professor \& Head \\ ${ }^{2}$ Assistant Professor \\ ${ }^{3}$ Professor \\ ${ }^{4}$ Senior Resident \\ ${ }^{5}$ Senior Resident \\ Department of E.N.T. \& Microbiology \\ Himalayan Institute of Medical Sciences \\ Jollygrant; Doiwala \\ DEHRADUN 248140 (Uttaranchal) \\ INDIA
}

Professor \& Head

Department of E.N.T

Himalayan Institute of Medical Sciences

Jollygrant; Doiwala

DEHRADUN - 248140 (Uttaranchal)

INDIA

E-mail: drsaurabh68@yahoo.com

\section{Case report}

A 15-years-old female was referred to the ENT Clinic of the Himalayan Institute Hospital, India, complaining of Right facial swelling, watering from right eye, progressive right nasal obstruction and intermittent nasal bleeding for more than 2 years. The patient had no history of pain, diminished vision, double vision, fever, or any symptom to suggest a systemic illness. On examination, patient was malnourished, her vital signs and general physical findings were normal. Patient had a cystic swelling 4x3 cm in right medial canthus area. On lacrimal syringing patient had post saccal stenosis and lacrimal swelling increased in size. Rest of the eye examination was normal. Rhinoscopy showed a fragile, mobile, and nontender polypoid mass in the right nasal fossa (endoscopic view of the nasal mass. Fig. 1), completely obstructing the nose. The color was red and its surface was irregular. The mass was attached to the septum and bled on contact. The nasal mucosa was hyperemic and swollen.Inferior turbinate and meatus of Rt. side could not be appreciated. The oropharynx also had a huge mass almost obliterating it. The results of routine laboratory tests were within normal limits. Coronal CT scan of the nose and paranasal sinuses revealed a cystic swelling, with airfluid level in lacrimal sac area (Fig. 2) (diagnosis of lacrimal diverticulum was made based on lacrimal syringing and CT scan), with an irregular mass filling the right nasal cavity completely, but no evidence of bony erosion with extension to the nasopharynx and oropharynx, with left sided deviated nasal septum and bilateral maxillary sinusitis (Fig. 3) Biopsy specimen from nasal mass was obtained under local anesthesia. Histopathological examination of the mass revealed Rhinosporidiosis. Complete surgical excision under general anaesthesia of the right lacrimal sac and nasal mass was done by lateral rhinotomy approach, removal of nasopharyngeal and oropharyngeal mass was done by pharyngeal route and cauterization of its base was performed. The intraoperative hemorrhage was negligible. The excised mass weighed 110 grams, was pink, with a 
Fig. 1 Clinical photograph (showing right medial canthal swelling, right nasal mass, oropharyngeal mass with left deviated nasal septum)

Fig. 2 Coronal ct scan showing right lacrimal diverticulum

Fig. 3 Coronal ct scan showing right nasal mass with left deviated nasal septum

Fig. 4 Histopathological examination of the nasal mass (H\&E '100)
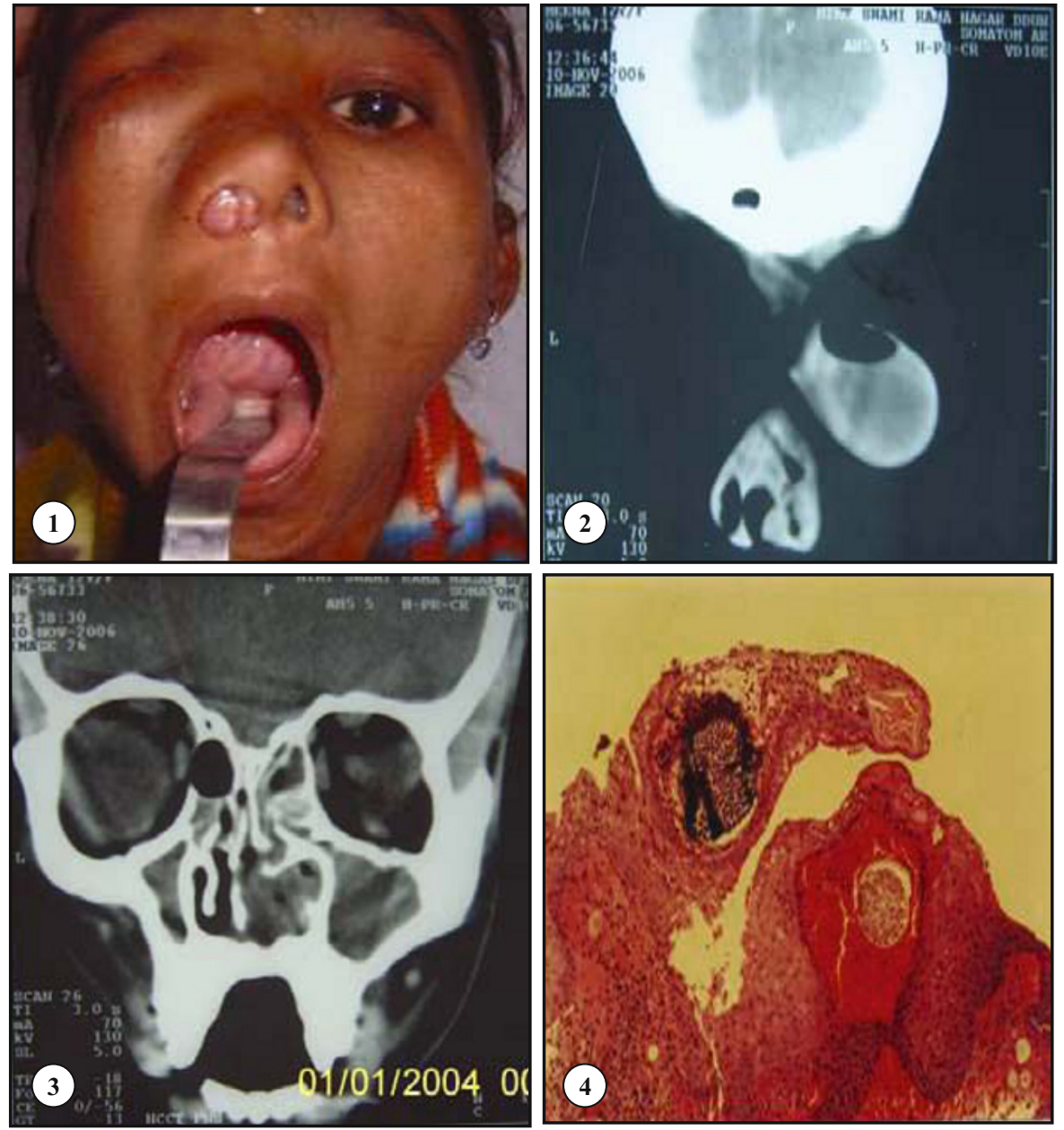

fleshy consistence, studded with whitish spots on its surface. On histological examination, the lesion showed the characteristic features of the rhinosporidiosis: the polypoid fibroconnective stroma, covered by flat multi-stratified squamous epithelium, contained many globular cysts. Each of these cysts represented a thick-walled sporangium containing numerous "daughter spores" in different stages of development (Fig. 4). The stroma contained a vascular fibroconnective tissue with fibroblasts and myofibroblasts and an inflammatory infiltrate (neutrophil granulocytes, lymphocytes, plasma cells and histiocytes). Histochemical stains such as PAS, GMS and mucicarmine were used to establish the correct diagnosis of rhinosporidiosis. Until today, after three months of follow-up, during which she underwent a clinical examination twice, she is healthy with no sign of recurrence.

\section{Discussion}

Rhinosporidiosis is a chronic granulomatous infection of the mucous membranes that usually manifests as vascular friable polyps that arise from the nasal mucosa or external structures of the eye. Initially described by Seeber in 1900, an individual from Argentina, rhinosporidiosis is endemic in India, Sri Lanka, South America, and Africa. Many cases from the United States and Southeast Asia, as well as scattered occurrences throughout the world, have been reported. Most cases of rhinosporidiosis occur in persons from or residing in the Indian subcontinent or Sri Lanka. Most persons with rhinosporidiosis have had bathing or working exposure to stagnant water. No known racial predilection exists. Men are affected more commonly than women, with a male-to-female ratio of $4: 1$. The disease most commonly occurs in children and in individuals aged 15-40 years

The etiologic agent, Rhinosporidium seeberi, has never been successfully propagated in vitro. Initially thought to be a parasite for more than 50 years, $R$ seeberi had been thought to be a water mold. Molecular biological techniques have more recently demonstrated that this organism is an aquatic protistan parasite. Recent $18 \mathrm{~S}$ ribosomal ribonucleic acid (rRNA) gene analysis has placed $R$ seeberi into a novel group of aquatic parasites of the class Mesomycetozoea. It is currently included in a new class, the Mesomycetozoea.

There is no demonstrated efficacy in using antifungal and/or antimicrobial drugs. Recurrence, dissemination in anatomical close sites and local secondary bacterial infections are the most frequent complications. 
Infection of the nose and nasopharynx is observed in $70 \%$ of persons with rhinosporidiosis; infection of the palpebral conjunctivae or associated structures (including the lacrimal apparatus) is observed in $15 \%$.

Other structures of the mouth and upper airway may be sites of disease. Disease of the skin, ear, genitals, and rectum has also been described. Genital disease has been described in the vagina, penile urethra or meatus, and scrotum. Dissemination of infection has been described in only 3 individuals.

Rhinosporidiosis is an infection that is typically limited to the mucosal epithelium. Infection usually results from a local traumatic inoculation with the organism. Disease progresses with the local replication of $R$ seeberi and associated hyperplastic growth of host tissue and a localized immune response. Rhinosporidiosis can cause prolonged painless disease with limited morbidity. Disease of up to 30 years' duration has been reported. Secondary bacterial infection can cause morbidity. Death has been reported only in the few rare reports of disseminated disease.

Nasal disease may present with unilateral nasal obstruction or epistaxis. Other symptoms may include local pruritus, coryza with sneezing, rhinorrhoea, and postnasal discharge with cough. Patients often report a sensation that a foreign body is present in their nasal cavity. Eye involvement initially is asymptomatic. Increased tearing may be reported as the disease progresses. Photophobia, redness, and secondary infection may occur. Skin lesions begin as papillomas that gradually become verrucous.

Soft polyps may be observed on the nose or eye. These polyps are pink to deep red, are sessile or pedunculated, and are often described as strawberrylike in appearance. Since the polyps of rhinosporidiosis are vascular and friable, they bleed easily upon manipulation.

Differential diagnosis with other problems such asNasal (allergic polyps, mucocele, malignancy), Ocular (hemangioma), Genital (condylomata, malignancy), Rectal (hemorrhoids), Cutaneous (warts) is to be considered.

Identifying the typical structures of R seeberi directly on microscopic examination makes diagnosis. This includes examination of smears of macerated tissue or histology of prepared biopsy sections. The organism can be observed with typical fungal stains (eg, Gomori methenamine silver [GMS], periodic acid-Schiff [PAS]), as well as with standard hematoxylin and eosin (H\&E) staining. Smears can also be observed with potassium hydroxide $(\mathrm{KOH})$ preparation.

Complications of the disease include extremely rare, life-threatening dissemination, local secondary bacterial infection, and recurrence. Prognosis is excellent, except with dissemination. Patients should be advised that recurrence is possible.

Diagnosis is based on history and clinical findings as well as histopathologic investigations. Nasal smears may be helpful as a screening test in suspected cases. No culture media for isolation of Rhinosporidium seeberi are available. Immuno-histochemical and electron-microscopic studies have also been done.

The treatment of choice is surgery. The best method is wide excision. The base of lesions must be cauterized to prevent recurrence of rhinosporidiosis. Dapsone and amphotericin have also been used, but with no benefit. Excessive bleeding, secondary bacterial infection, and fatal sepsis are mentioned as the complications of treatment.

\section{Summary}

Rhinosporidiosis is a chronic disease caused by a funguslike microorganism, known as Rhinosporidium seeberi. Histopathologically, round submucosal cysts containing spores are formed. They are called sporangia. The sporangia are diagnostic Nasal mucosa is involved in $72 \%$ of the cases. The nasal lesion presents as a painless polypoid mass with a dull pink or red color. Epistaxis and nasal obstruction are the most common findings. It may be unilateral or bilateral. The enlargement of these lesions occurs slowly over months or years. A case of Rhinosporidiosis with an unusual feature of origin in the lacrimal sac with spread to the naso-maxillary area and chronicity of 22 years has been reported. Rhinosporidiosis mainly occurs in India and Sri Lanka as an endemic disease, but sporadic cases from other parts of the world have also been reported. It has been reported from the USA, Canada, Russia, Scotland, England, Italy, Brazil, Cuba, Mexico, Argentina, Paraguay, Ecuador, Africa, Indonesia, Malaysia, Philippines, Japan, as well as Iran. Rhinosporidiosis is most often seen in young males, but it may be found at any age from 3 to 90 years in both sexes. The disease is found in all socio-economic strata and among all communities and persons belonging to different religious groups. Most of the patients gave a history of bathing in muddy stagnant pools of water. The mode of infection is not clear, but immersion in contaminated waters in Asia and Africa and dusts from the dung of infected horses and cattle are mentioned as the possible responsible factors. Rhinosporidiosis of the eye (conjunctiva and lacrimal sac), maxillary sinuses, nasopharynx, epiglottis, larynx, trachea, bronchus, ear, scalp, skin, urethra, and external genitalia are occasionally reported. Very few cases of diverticulum of the lacrimal sac associated with rhinosporidiosis are reported A high incidence of ocular rhinosporidiosis in Kanyakumari district of Tamil Nadu, India is reported.

\section{References}

1. Vincent Y, Gerard M, Ermens F, Depierreux M (1982) Tumor of the nasal cavity unusual in our region. Apropos of a case of rhinosporidiosis [in French]. Acta Otorhino-laryngol Belg 36: 1021-1028

2. Jaiswal V, Kumar M, Gupta S, Kherdekar M, Mahore MN (1992) Cytodiagnosis of rhinosporidiosis. J Trop Med Hyg 95:71-72 
3. MM Krishnan, VK Kawatra, VA Rao and C Ratnakar (1986) Diverticulum of the lacrimal sac associated with rhinosporidiosis. British Journal of Ophthalmology 70:867-868

4. Rao NS, Prasad DLN, Reddy DVR (1994) Rhinosporidiosis - a case report. Indian Journal of Plastic Surgery 27 (1): $31-32$

5. J. S. Moses, C. Balachandran, S. Sandhanam, N. Ratnasamy, S. Thanappan, Johnson Rajaswar, Dinakar Moses (1990) Ocular rhinosporidiosis in Tamil Nadu, India. Mycopathologia 111(1):5-8

6. Luca Morelli, Mario Polce, Francesco Piscioli, Franca Del Nonno, Renato Covello, Alessia Brenna, Antonio Cione, Stefano Licci (2006) Human nasal rhinosporidiosis: an Italian case report. Diagn Pathol 1:25
7. Mona Harissi-Dagher, Nicole Robillard, Christine Corriveau, Michèle Mabon, Guy S. Allaire (2006) Histopathologically confirmed ocular rhinosporidiosis in two Canadians. Canadian Journal of Ophthalmology 41(2):226-229

8. Fredricks DN, Jolley JA, Lepp PW, et al (2000) Rhinosporidium seeberi: a human pathogen from a novel group of aquatic protistan parasites. Emerg Infect Dis 6(3):273-282

9. Mohan H, Chander J, Dhir R, Singhal U, Mendoza L, Taylor JW, Ajello L (1995) The class mesomycetozoea: a heterogeneous group of microorganisms at Rhinosporidiosis in India: a case report and review of literature. Mycoses 38(5-6):223-225

10. Job A, Venkateswaran S, Mathan M, et al (1993) Medical therapy of rhinosporidiosis with dapsone. J Laryngol Otol 107(9):809-812 